\title{
Improving Identification of Food-Insecure Patients in an Outpatient Clinic Setting
}

\author{
Oluwaseun O. Acquah, MD | Amanda M. Honsvall Hoefler, MD | Isaac Zoller, MD | Lynn P. \\ Manning, MD | Donald J. Pine, MD | Richard F. Mitchell, MD, MS
}

PRiMER. 2020;4:3.

Published: 2/21/2020 | DOI: 10.22454/PRiMER.2020.115304

\section{Abstract}

Introduction: Food insecurity $(\mathrm{FI})$ is defined as limited or uncertain access to enough nutritious food for all household members to lead an active and healthy life. In 2017 , roughly $12 \%$ of US households reported FI. FI screening is not standard practice despite Fl's association with poor health outcomes. This study compared FI screening strategies in a community-based family medicine residency clinic to determine which strategies identified the largest number of FI patients.

Methods: We conducted this study using a validated two-question screening tool with high sensitivity and specificity for identifying FI. Three implementation strategies of the screening tool were tested: two clinicianinitiated and one staff-initiated. Data measured included opportunities to screen, patients actually screened, and the number of positive (disclosure) responses.

Results: Clinician-initiated screening rates increased when clinicians followed a standard note template with embedded FI questionnaire vs no template ( $58.6 \%$ vs $7.1 \%$ ). Despite this improvement, staff-initiated screening returned an even higher screening rate (95.2\%). The disclosure of FI determined by staff-initiated screening was also higher (12.2\%, similar to previously published data) than clinician-initiated screening $(2.3 \%)$.

Conclusions: Staff-initiated screening for FI was the best way of identifying FI patients and yielded results consistent with local and national estimates. Clinicians did not screen patients for Fl often enough for this approach to be effective, but embedding FI screening into templated notes improved clinician screening rates. Disclosure of $\mathrm{Fl}$ when staff conducted screening far exceeded disclosure when screening was initiated by clinicians.

\section{Introduction}

Food insecurity (FI) is defined as "limited or uncertain availability of nutritionally adequate and safe foods, or limited or uncertain ability to acquire acceptable foods in socially acceptable ways." ${ }^{11} \mathrm{Fl}$ is associated with adverse health outcomes. Even when controlling for confounders such as income and housing instability, Fl is associated with high rates of obesity and diet-related chronic disease. ${ }^{2} \mathrm{Fl}$ is particularly harmful for youth, predisposing children to poorer general health, more frequent hospitalizations, slower recovery from illness, poorer academic performance, and higher levels of behavioral and emotional problems from preschool through adolescence. ${ }^{3}$

According to the United States Department of Agriculture, $11.8 \%$ of households and $15.7 \%$ of households with children reported $\mathrm{Fl}$ in $2017 .{ }^{4}$ In 2014 , only $58 \%$ of food insecure households participated in one or more of three federal assistance programs, despite the programs' role in alleviating poverty, $\mathrm{FI}$, and improving well-being. ${ }^{4,5}$ In 
Hennepin County, Minnesota, $12 \%$ of all people are estimated to be food insecure. Our clinic population is demographically similar to the county as a whole in age breakdown, gender, and race/ethnicity (ie, $68 \%$ Caucasian, $18 \%$ African-American, $6.5 \%$ Hispanic in our clinic, vs $68 \%$ Caucasian, $13.6 \%$ African-American, and $7.0 \%$ Hispanic in Hennepin County) ${ }^{5,8,9}$ Despite the role of $\mathrm{FI}$ as a social determinant of health (SDH), Fl screening is currently not standard practice. Our objective was to test clinic processes for Fl screening to determine which method would identify the largest number of patients who could benefit from FI intervention.

\section{Methods}

We conducted the study using a validated two-question screening tool ( $97 \%$ sensitivity, $83 \%$ specificity for $\mathrm{Fl}$ ), where a positive screen is defined as a "yes" response to at least one of the two questions (Figure 1). 3,7 We conducted the study in a community-based family medicine residency clinic between 2015 and 2018. We adjusted our workflow serially to optimize the number of Fl patients identified. In methods 1 and 2, clinicians could either opt in or opt out of asking patients the screening questions during well exams (Table 1). In method 3 , frontline staff gave each patient a card with the screening questions during check in, and nursing staff collected the cards while rooming patients. If a patient did not record answers on the card, they were still counted in the number of screening opportunities, but not in the number of completed screens.

Table 1 describes the different methods tested, each lasting 4 to 6 weeks. Data collected included the number of patient encounters where screening could occur, the number where patients were screened, and the number of positive screens. We calculated screening rates as the number of completed screens vs opportunities (Figure 2), and we calculated disclosure rates as the number of positive screens vs completed screens (Figure 3 ).

A single-site convenience sample was used, and our Fl team consisted of the following members with their approximate numbers in parentheses: residents (18), faculty (11-12), frontline staff (3-4), nursing (3-4), social work (3), care coordinator (1), and clinic management (1). There was variation in the individual participants from method to method. Residents led the development and design of each screening trial. All team members received education on Fl before and during the study. Our social work/care coordinator team met with patients who disclosed FI and assessed eligibility and access to federal resources as well as local food shelves. Food boxes were supplied for patients with immediate need for food. The Park Nicollet Institutional Review Board reviewed this study and determined it to be quality improvement (QI), and exempt from further oversight.

\section{Results}

When clinicians decided who they should screen for food insecurity during well exams, the screening rate was only $7.1 \%$. This increased to $58.6 \%$ when clinicians were asked to screen by default and needed to delete the question from a standard note template to opt out. The actual number of identified patients in both cases was very low (only one patient identified in each case). Having staff initiate the screening process and collect results led to a much higher screening rate (95.2\%; Table 2, Figure 2 ) and to a disclosure rate that was close to the reported prevalence for Hennepin County (12.2\% vs $12 \%)$ and the United States (11.8\%; Figure 3 ).

\section{Conclusions}

Clinician-initiated screening yielded a substantially lower disclosure rate than staff-initiated screening, suggesting that the former method is inferior at identifying patients who struggle with FI. A possible reason for this is that clinicians only screened for FI during well-exam visit types, significantly limiting screening opportunities. Our data also support the conclusion that the FI screening rate is higher when clinicians are prompted to screen by a templated note in an EMR from which they need to opt out (58.6\% vs 7.1\%; Table 2). This approach may effectively increase clinician screening rates but may not increase patient disclosure rates.

It is unclear why patients disclose Fl less often to clinicians than to staff, but we suspect that social desirability bias may be present. Social desirability bias could also explain why only $58 \%$ of food insecure families participated in 
one of the three federal programs alluded to in the introduction. While patients were not observed while completing the screen when staff initiated screening, for the clinician-initiated screening clinicians asked patients about FI directly during the first two methods. This direct questioning may have increased patients' reluctance to disclose. Clinician discomfort addressing a patient's socioeconomic status may lead to missed screening opportunities and may also drive patient reluctance to disclose $\mathrm{Fl}$.

The estimated prevalence of $\mathrm{Fl}$, based on the disclosure rate in our population (12.2\%), is consistent with the estimated prevalence of FI in Hennepin County, Minnesota (12\%), and in the United States (11.8\%; Figure 2). One limitation of our study is the possibility of overestimates in disclosure rates. Since a card was filled out for each patient encounter, not for each unique patient, some patients with FI could have been counted twice. However, the number of duplicate reports is likely to be small, since each period of inquiry was about 1 month long, and a followup visit during that time period is less likely. Finally, this was a single-site study, and as such may not be generalizable to other settings.

We anticipate following our FI patient population to determine if interventions by our social work team reduced $\mathrm{FI}$ and to evaluate whether reduced Fl leads to improved health. It is beyond the scope of this article to address the ongoing debate on screening for social determinants of health in the clinic, but we hope that this study is useful to others attempting to improve their Fl screening process. Future study and coordination with community resources is essential for understanding how best to help some of our most vulnerable patients. ${ }^{10}$

\section{Tables and Figures}

Figure 1: USDA-Validated Two-Question Food Insecurity Screen

Food insecurity, or lack of food, can mean skipping meals to make food last longer. It can also mean having to choose between buying food or paying your bills. We can help if you and your family are dealing with this.

1. Within the past 12 months, have you ever worried that your food would run out before you got money to buy more?

Yes $\square \quad$ No

2. Within the past 12 months, did the food that you bought just didn't last and you didn't have enough money to get more?

Yes $\square \quad$ No

Table 1: Description of the Three Screening Methods

\begin{tabular}{|c|l|l|l|}
\hline Method & \multicolumn{1}{|c|}{ Type } & \multicolumn{1}{c|}{$\begin{array}{c}\text { When } \\
\text { Conducted }\end{array}$} & Description \\
\hline 1 & $\begin{array}{l}\text { Clinician } \\
\text { initiated: } \\
\text { opt in }\end{array}$ & June 2015 & $\begin{array}{l}\text { Clinicians were encouraged to opt in by completing the questionnaire during well } \\
\text { exams. They could choose to paste a standardized template into their note to } \\
\text { serve as both script and documentation of the Fl screen. }\end{array}$ \\
\hline 2 & $\begin{array}{l}\text { Clinician } \\
\text { initiated: } \\
\text { opt out }\end{array}$ & $\begin{array}{l}\text { November } \\
2015\end{array}$ & $\begin{array}{l}\text { The Fl questionnaire was embedded into the well exam template for clinicians to } \\
\text { complete during well exams. Clinicians had the option to delete the questionnaire } \\
\text { from the template. }\end{array}$ \\
\hline 3 & Staff initiated & $\begin{array}{l}\text { June 2017, } \\
\text { November } \\
2017, \text { June } \\
2018\end{array}$ & $\begin{array}{l}\text { A card containing the questionnaire was offered to all patients at check in. Cards } \\
\text { were collected during rooming by nursing staff. An identification sticker was } \\
\text { placed on the card so patients could be contacted or seen that same day by a } \\
\text { social worker. All cards offered were counted as screening opportunities; those } \\
\text { that were marked were further counted as completed screens. }\end{array}$ \\
\hline
\end{tabular}



Figure 2: Screening Rates (Screens Collected vs Opportunities
to Screen) for Different Screening Methods

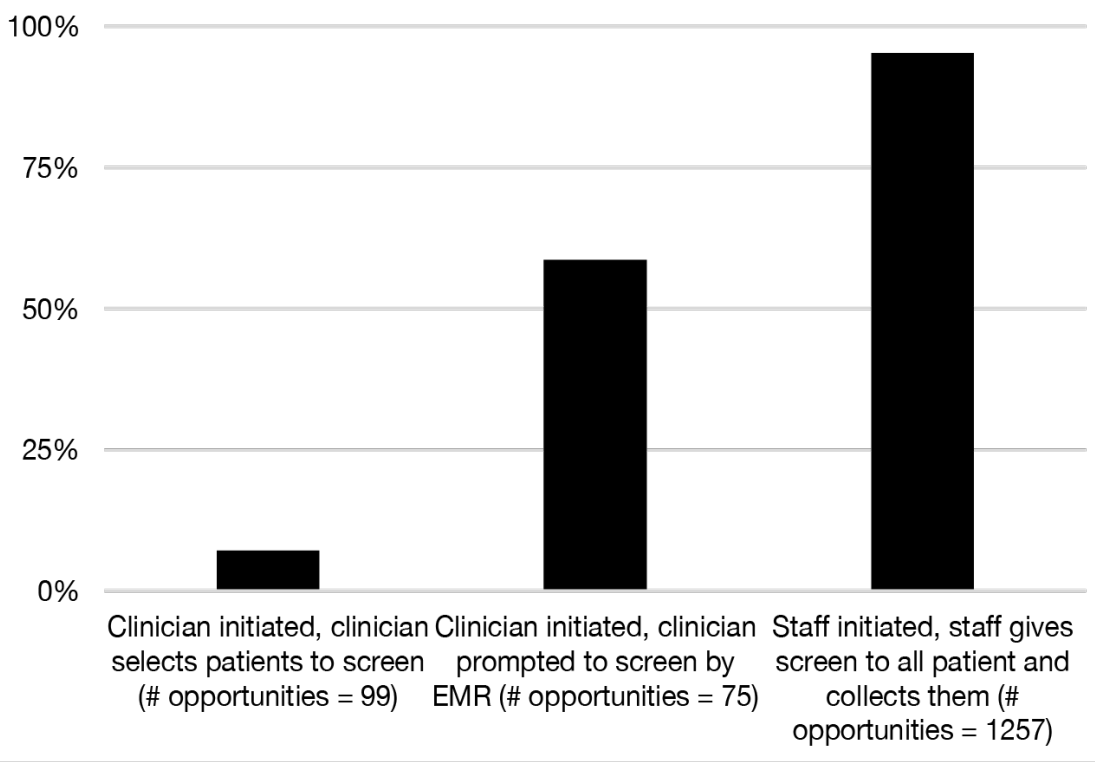

Figure 3: Food Insecurity Disclosure Rates

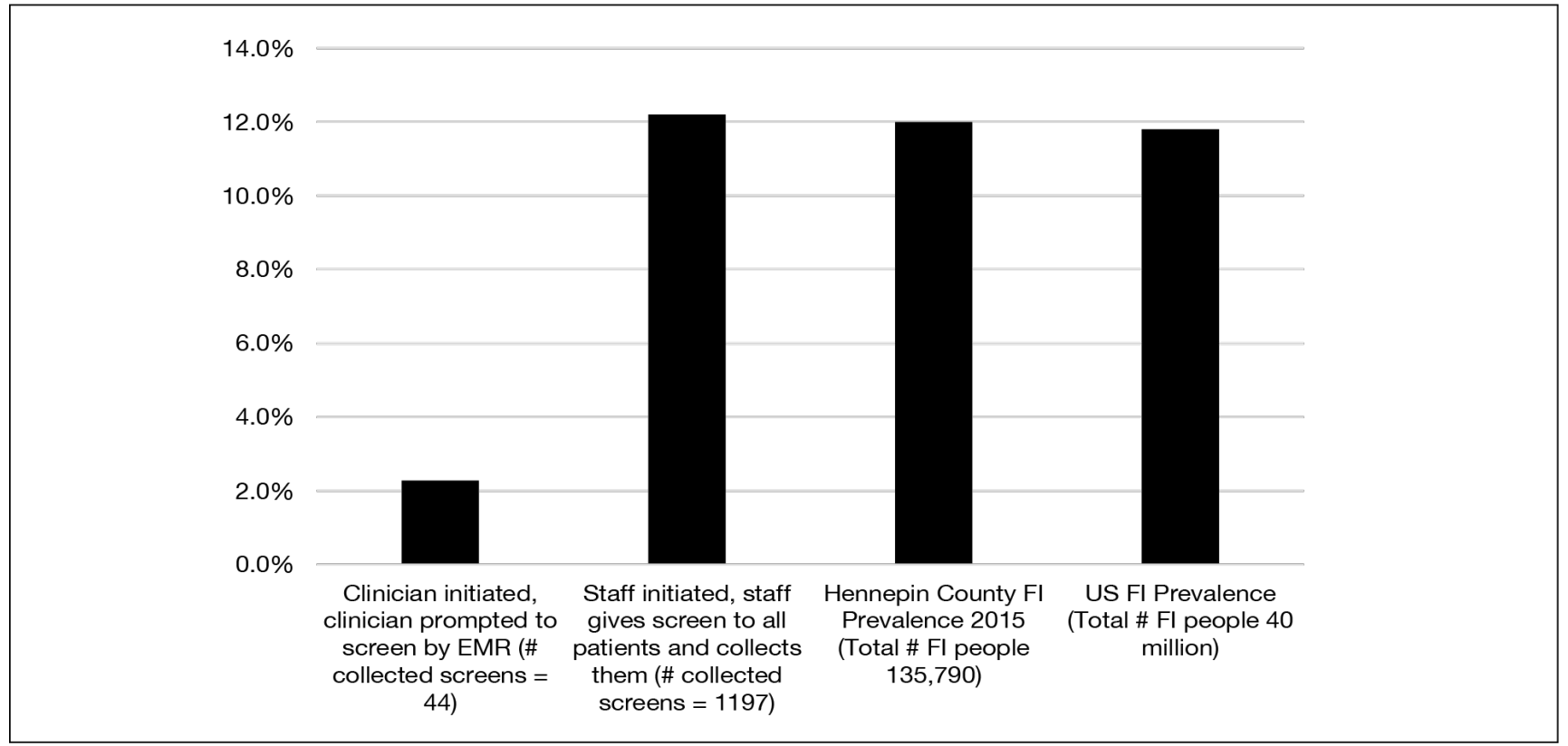

Table 2: Results From the Three Screening Methods

\begin{tabular}{|c|c|c|c|c|c|c|}
\hline Phase & Type & $\begin{array}{c}\text { Opportunities to } \\
\text { Screen (Opp) }\end{array}$ & $\begin{array}{c}\text { Completed } \\
\text { Screens } \\
\text { (Com) }\end{array}$ & $\begin{array}{c}\text { Positive } \\
\text { Screens } \\
\text { (Pos) }\end{array}$ & $\begin{array}{c}\text { Screening } \\
\text { Rate (Com/ } \\
\text { Opp \%) }\end{array}$ & $\begin{array}{c}\text { Disclosure Rate } \\
\text { (Pos/Com\%) }\end{array}$ \\
\hline 1 & $\begin{array}{c}\text { Clinician initiated: } \\
\text { opt in }\end{array}$ & 99 (only well exams) & 7 & 1 & 7.1 & $\begin{array}{c}\mathrm{n} / \mathrm{a}(\text { (sample size too } \\
\text { small) }\end{array}$ \\
\hline 2 & $\begin{array}{c}\text { Clinician initiated: } \\
\text { opt out }\end{array}$ & 75 (only well exams) & 44 & 1 & 58.6 & 2.3 \\
\hline 3 & Staff initiated & 1,257 (all visits) & 1,197 & 146 & 95.2 & 12.2 \\
\hline
\end{tabular}




\section{Acknowledgments}

The authors thank the following individuals for their assistance in the development, coordination and execution of their research: Allyson Hayward, MSW, LICSW; Alice Macdonald, MBChB; Judith Boylan, MD; Ashley Estrem, DO; Kasey Kapella, MD; Deborah Mullen, PhD; and Beth Pearson, RN.

Financial Support: A January 2016 Minnesota Academy of Pediatrics grant helped this work to continue in collaboration with three other practices in the Twin Cities. As part of this grant, residents attended and were actively engaged in a series of the University of Minnesota Extension's Supplemental Nutrition Assistance Program Education (SNAP-Ed) cooking classes for our patients and their families.

Presentations: This study was presented at the following conferences:

- AAFP FMX Conference, September 2016, Orlando, FL

- MAFP Research and Innovation Forum, April 2017, Bloomington, MN

- AAFP National Conference for Residents and Medical Students, July 2017, Kansas City, MO (Winning poster)

- AAFP FMX Conference, September 2017, San Antonio, TX

- STFM Annual Spring Conference, May 2018, Washington, DC

- MMCGME Annual Resident \& Fellow Quality Forum, May 2018, University of Minnesota, Minneapolis, MN (Winning Poster)

- MMA Annual Conference, September 2018, St Paul, MN (Winning poster)

- STFM Conference on Practice Improvement, December 2018, Tampa, FL (H. Winter Griffith Scholarship winner)

\section{Corresponding Author}

Oluwaseun O. Acquah, MD

University of Minnesota Methodist Hospital Family Medicine Residency Program, 6600 Excelsior Blvd, Suite 160, St Louis Park, MN 55426. 952-993-7711. Fax: 952-993-6798.

oluwaseun.acquah@parknicollet.com

\section{Author Affiliations}

Oluwaseun O. Acquah, MD - University of Minnesota Methodist Hospital Family Medicine Residency Program, St Louis Park, MN

Amanda M. Honsvall Hoefler, MD - University of Minnesota Methodist Hospital Family Medicine Residency Program, St Louis Park, MN

Isaac Zoller, MD - University of Minnesota Methodist Hospital Family Medicine Residency Program, St Louis Park, $\mathrm{MN}$

Lynn P. Manning, MD - University of Minnesota Methodist Hospital Family Medicine Residency Program, St Louis Park, MN

Donald J. Pine, MD - University of Minnesota Methodist Hospital Family Medicine Residency Program, St Louis Park, MN

Richard F. Mitchell, MD, MS - University of Minnesota Methodist Hospital Family Medicine Residency, St Louis Park, $\mathrm{MN}$

\section{References}

1. Andersen SA. Core indicators of nutritional state for difficult-to-sample populations. J Nutr. 1990;120(suppl 11):1559-1600. https://doi.org/10.1093/jn/120.suppl_11.1555

2. Hartline-Grafton H, Dean O. Hunger \& Health: The Impact of Poverty, Food Insecurity, and Poor Nutrition on Health and Well-Being. Washington, DC: Food Research \& Action Center; December 2017. http://www. frac.org /wp-content/uploads/hunger-health-impact-poverty-food-insecurity-health-well-being.pdf. Accessed February 11, 2019. 
3. Council on Community Pediatrics, Committee on Nutrition. Promoting food security for all children.

Pediatrics. 2015;136(5):e1431-e1438. https://doi.org/10.1542/peds.2015-3301

4. Coleman-Jensen A, Rabbitt MP, Gregory CA, Singh A. Household Food Security in the United States in 2017. United States Department of Agriculture Economic Research Service: Economic Research Report \#256. September 2018.

5. Hartline-Grafton, H. Hunger \& Health: The Role of the Supplemental Nutrition Assistance Program in Improving Health and Well-Being. Washington, DC: Food Research \& Action Center; 2017. http://www.frac.org /research/resource-library. Accessed February 17, 2020.

6. County Health Rankings \& Roadmaps (CHR\&R). Minnesota: 2015 Ranking for Food Insecurity Measure. https://www.countyhealthrankings.org/app/minnesota/2015/measure/factors/139/data. Accessed August 8, 2019.

7. Hager ER, Quigg AM, Black MM, et al. Development and validity of a 2-item screen to identify families at risk for food insecurity. Pediatrics. 2010;126(1):e26-e32. https://doi.org/10.1542/peds.2009-3146

8. United States Census Bureau. QuickFacts: Hennepin County, MN. https://www.census.gov/quickfacts /fact/table/hennepincountyminnesota/PST045218. Accessed August 1, 2019.

9. Internal data, Park Nicollet Clinic - Creekside. Accessed via database query July, 2019.

10. Davidson KW, McGinn T. Screening for Social Determinants of Health: The Known and Unknown. JAMA. 2019;322(11):1037-1038. https://doi.org/10.1001/jama.2019.10915

Copyright $₫ 2020$ by the Society of Teachers of Family Medicine 